\title{
The Effects of Scaffold Remnants in Decellularized Tissue-Engineered Cardiovascular Constructs on the Recruitment of Blood Cells
}

\author{
Bart Sanders, PhD, ${ }^{1,2}$ Anita Driessen-Mol, PhD, ${ }^{1,2}$ Carlijn V.C. Bouten, PhD, ${ }^{1,2}$ and Frank P.T. Baaijens, PhD ${ }^{1,2}$
}

Decellularized tissue-engineered heart valves (DTEHVs) showed remarkable results in translational animal models, leading to recellularization within hours after implantation. This is crucial to enable tissue remodeling. To investigate if the presence of scaffold remnants before implantation is responsible for the fast recellularization of DTEHVs, an in vitro mesofluidic system was used. Human granulocyte and agranulocyte fractions were isolated, stained, brought back in suspension, and implemented in the system. Three different types of biomaterials were exposed to the circulating blood cells, consisting of decellularized tissue-engineered constructs (DTECs) with or without scaffold remnants or only bare scaffold. After $5 \mathrm{~h}$ of testing, the granulocyte fraction depleted faster from the circulation than the agranulocyte fraction. However, only granulocytes infiltrated into the DTEC with scaffold, migrating toward the scaffold remnants. The agranulocyte population, on the other hand, was only observed on the outer surface. Active cell infiltration was associated with increased levels of matrix metalloproteinase-1 secretion in the DTEC, including scaffold remnants. Proinflammatory cytokines such as interleukin (IL)-1 $\alpha$, IL-6, and tumor necrosis factor alpha $(\mathrm{TNF} \alpha)$ were significantly upregulated in the DTEC without scaffold remnants. These results indicate that scaffold remnants can influence the immune response in DTEC, being responsible for rapid cell infiltration.

Keywords: immune response, blood cells, scaffold, decellularization, tissue engineering, heart valves

\section{Introduction}

C ONGENITAL HEART DISEASES, including heart valve defects, affect 1.35 million newborns annually, making them accountable for one-third of all major birth defects. ${ }^{1}$ Currently, pediatric patients who undergo heart valve replacement require multiple surgical interventions since none of the current available prostheses can accommodate for growth, repair, and remodeling. ${ }^{2}$ This is associated with increasing rates of morbidity and mortality. ${ }^{3}$

Over the years, various decellularized bioprostheses have been investigated for their potential to provide heart valve implants with regenerative capacity. ${ }^{4}$ Recellularization is of crucial importance to rearrange the extracellular matrix and facilitate future growth. However, xenografts ${ }^{5}$ or allografts 6 have so far shown little evidence of proper recellularization by the host. ${ }^{7}$ This limitation can be overcome by the use of tissue-engineered implants. ${ }^{8}$

Based on our recent in vivo studies, in which decellularized tissue-engineered heart valves (DTEHVs) were implanted in sheep and nonhuman primates, recellularization with a first influx of blood-derived cells was observed within hours of implantation. Furthermore, a secondary influx of cells from the adjacent native tissue gradually started to infiltrate toward the leaflets within 8 weeks. ${ }^{9,10}$ However, the mechanism that triggers the cellular influx is still unknown. Typically, infiltrated cells group in the vicinity of scaffold remnants. Therefore, it is hypothesized that the presence of scaffold remnants might trigger the first influx of cells.

In addition, the response of immune cells is known to differ between biomaterials and may play a critical role in guiding the regenerative response. ${ }^{11,12}$ Depending on the cytokine

\footnotetext{
${ }^{1}$ Department of Biomedical Engineering, Eindhoven University of Technology, Eindhoven, The Netherlands.

${ }^{2}$ Institute for Complex Molecular Systems, Eindhoven University of Technology, Eindhoven, The Netherlands.

(c) Bart Sanders et al. 2017; Published by Mary Ann Liebert, Inc. This Open Access article is distributed under the terms of the Creative Commons Attribution Noncommercial License (http://creativecommons.org/licenses/by-nc/4.0/) which permits any noncommercial use, distribution, and reproduction in any medium, provided the original author(s) and the source are credited.

*This article is part of a special focus issue on Strategic Directions in Immunoresponsive Biomaterials in Tissue Engineering. An additional article can be found in Tissue Engineering Part B, volume 23, number 5.
} 
environment created by the first influx of cells, polarization, for instance, of t-helper cells and macrophages, can be affected and influence tissue regeneration. ${ }^{13}$

To investigate if scaffold remnants trigger the first cellular influx and to elucidate on the nature of the immune response, a mesofluidic in vitro flow setup was used. ${ }^{14}$ This platform consists of a cross-flow chamber incorporated into a flow loop, where the sample of interest is exposed to circulating blood cells under physiological wall shear stress and perfusion pressure. This system creates a fully developed flow profile with homogeneous shear stress distribution over the sample.

Decellularized tissue-engineered constructs (DTECs) with and without scaffold remnants were included, as well as bare scaffold samples. Granulocyte and agranulocyte fractions were isolated from human peripheral blood, stained, brought back in suspension, and incorporated in the system. Every hour, the composition of the remaining circulating cell population was analyzed through flow cytometry, where the experiment was terminated after $5 \mathrm{~h}$. Samples from the circulation were taken for enzyme-linked immunosorbent assay (ELISA) to investigate the released cytokine profiles. The biomaterials were subsequently subjected to scanning electron microscopy (SEM) for surface analyses, confocal microscopy, and fluorescence microscopy for cellular infiltration.

\section{Materials and Methods}

\section{Biomaterials}

DTECs were produced and analyzed according to previously developed protocols. ${ }^{15}$ Within this study, three different biomaterials were evaluated under equivalent conditions. One group contained only scaffold, a second group contained tissue and scaffold remnants, and a third group contained only tissue.

Construct 1: bare scaffold. Before their use in the mesofluidic setup, circular scaffolds $(n=4)$ with a diameter of $2.5 \mathrm{~cm}$ were cut from nonwoven polyglycolic acid meshes (PGA, thickness $1.0 \mathrm{~mm}$, specific gravity $70 \mathrm{mg} / \mathrm{cm}^{3}$; Cellon, Luxembourg) (Fig. 1A).
Construct 2: DTECs with scaffold. Circular PGA scaffolds $(n=4)$ were sewn (Prolene 4-0; Ethicon) onto stainless steel rings, coated with $1 \%$ poly-4-hydroxybutyrate (P4HB; MW: $1 \times 10^{6}$; TEPHA, Inc.) in tetrahydrofuran (THF; Sigma-Aldrich), placed overnight in a six-well plate to evaporate the THF, and cultured as described below before use in the mesofluidic setup (Fig. 1B).

Construct 3: DTECs without scaffold. DTECs without scaffold $(n=4)$ were grown in circular-shaped Velcro rings with a diameter of $2.5 \mathrm{~cm}$ based on the use of fibrin. The Velcro parts were mounted (Silastic MDX4-4210; Dow Corning) into the bottom of six-well plates and cured overnight at $37^{\circ} \mathrm{C}$. The patches were cultured as described below and placed in the mesofluidic setup (Fig. 1C).

Tissue culture. Sterilization of biomaterials was performed by immersion in 70\% ethanol (EtOH; VWR International S.A.S. Fontenay-Sous-Bois, France) for $15 \mathrm{~min}$. Thereafter, the samples were washed three times with phosphate-buffered saline (PBS) for $10 \mathrm{~min}$, incubated in an antibacterial-antifungal solution, $10 \%$ penicillin/streptomycin (Pen/Strep) (Lonza, Belgium), supplemented with 0.5\% Fungin (InvivoGen) for $30 \mathrm{~min}$, and washed three times with PBS for $10 \mathrm{~min}$. Hereafter, the constructs were incubated overnight in growth medium (Advanced Dulbecco's modified Eagle's medium [ADMEM; Gibco], supplemented with 10\% fetal bovine serum [FBS; Biochrom, Germany], 1\% Pen/ Strep, and 1\% Glutamax [Gibco]).

Primary isolated human vascular-derived venous myofibroblasts were harvested from the vena saphena magna of a 77 -year-old patient, ${ }^{16}$ according to the Dutch guidelines for secondary used materials $\left(0.3 \times 106 \mathrm{cells} / \mathrm{cm}^{2}\right.$, passage 6$)$. The tissue production capacity of these cells has been thoroughly investigated in previous studies, confirming mature extracellular matrix formation, comprising densely packed collagen and glycosaminoglycans. ${ }^{10,15,17,18}$ The cells were seeded either into the PGA scaffold or into the Velcro rings, using fibrin as a cell carrier. The seeded constructs were provided with growth medium supplemented with L-ascorbic

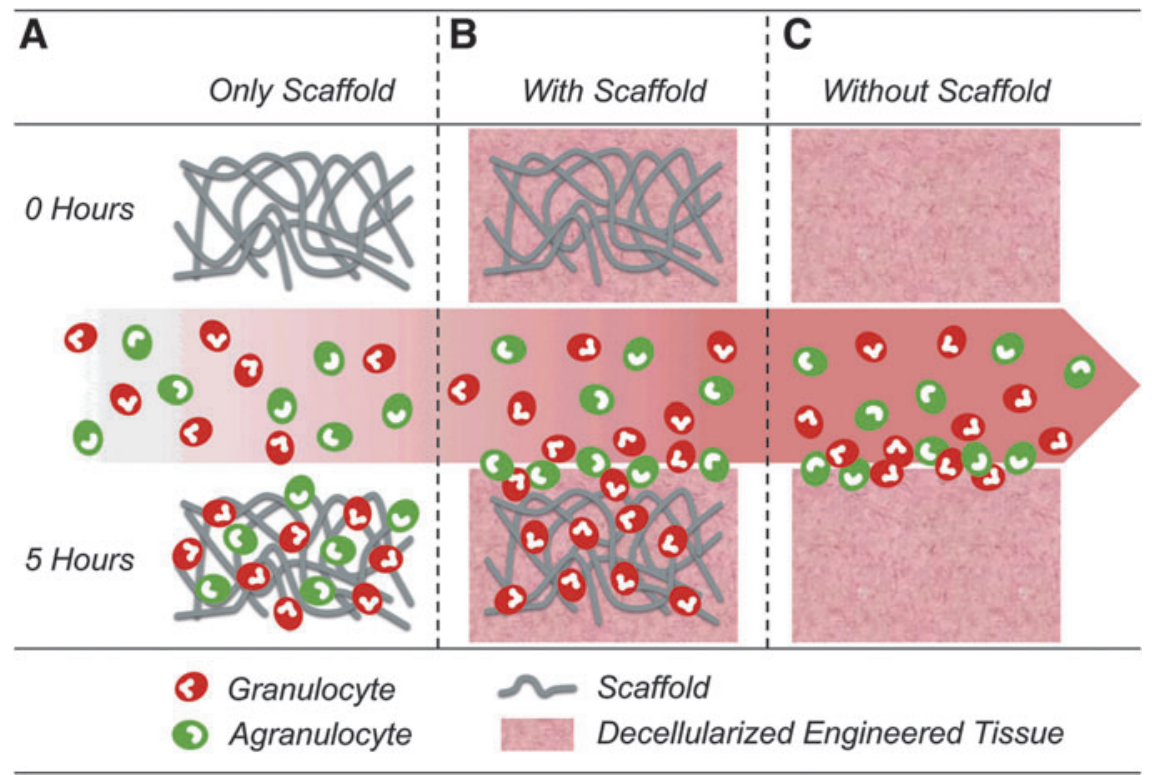

FIG. 1. Schematic overview. Biomaterials of interest used in this study, with bare scaffold (A), decellularized tissue-engineered construct with scaffold remnants (B), and decellularized tissue-engineered constructs without scaffold remnants $(\mathbf{C})$. Before the experiment, all constructs were acellular. The circulating cell population contained the agranulocyte fraction in green and the granulocyte fraction in red. After $5 \mathrm{~h}$ of the experiment, the bare scaffold material contained both cell types, the tissue with scaffold remnants contained only the granulocyte fraction and the tissue without scaffold remnants only revealed a surface layer of cells. 
acid 2-phosphate $(0.25 \mathrm{mg} / \mathrm{mL}$; Sigma $)$ and 6-aminocaproic acid (ACA, $1 \mathrm{mg} / \mathrm{mL}$; Sigma) for 2 weeks, whereafter the ACA was removed from the medium and patches were cultured for additional 2 weeks.

Decellularization. After 4 weeks of culture, all tissueengineered patches $(n=8)$, both with and without scaffold, were decellularized as previously described. ${ }^{19}$ Briefly, the patches were washed three times for $10 \mathrm{~min}$ with PBS and decellularized overnight in detergent solution $(0.25 \%$ Triton $\mathrm{X}-100$, sodium deoxycholate, and $0.02 \%$ EDTA). Nucleic remnants were enzymatically removed by using three Benzonase (EMD Millipore) incubation steps and diluted in $50 \mathrm{mM}$ TRIS-HCL buffer solution in concentrations of 100 , 80 , and $20 \mathrm{U} / \mathrm{mL}$ for 8,16 , and $8 \mathrm{~h}$, respectively, on a shaker at $37^{\circ} \mathrm{C}$. Afterward, the patches were washed three times with PBS and incubated for $24 \mathrm{~h}$ in M-199 medium (Gibco) on a shaker at $4^{\circ} \mathrm{C}$ to remove cellular remnants. Patches were washed three times with PBS, sterilized with $70 \% \mathrm{EtOH}$ for $15 \mathrm{~min}$, washed three times with PBS, and incubated for $30 \mathrm{~min}$ in an antibacterial-antifungal solution. After sterilization, patches were stored at $4^{\circ} \mathrm{C}$ until further use.

\section{Experimental setup}

Blood collection and preparation. Peripheral blood samples $(40 \mathrm{~mL})$ were isolated from healthy human male donors ( $n=4$ with age between 26 and 32) under informed consent, as approved by the Dutch Medical research Ethics Committees United, and registered with reference name "Initial Immune Response to new Biomaterials" and number NL53574.100.15. The blood samples were separated into fractions of plasma, agranulocytes, granulocytes, and erythrocytes through density gradient centrifuging at $700 \mathrm{~g}$ over two stacked layers of histopague with different densities (Histopague 1119 and Histopague 1077; Sigma-Aldrich) and washed with PBS. The isolated agranulocyte fraction was stained with CellTracker Green (Life Technologies) and the granulocyte fraction with CellTracker Orange (Life Technologies) for $30 \mathrm{~min}$ and washed with PBS. Erythrocytes were discarded, whereafter the stained granulocyte and agranulocyte fractions were resuspended in the plasma and added up to $40 \mathrm{~mL}$ with PBS.

Mesofluidic system. An in-house developed mesofluidic setup was used to investigate the interaction of circulating cells with biomaterials as described by Smits et al. ${ }^{14}$ In brief, rectangular strips $\left(10 \times 15 \mathrm{~mm}^{2}\right)$ were taken from the individual biomaterials and positioned into three independent fluidic units (Ibidi $\mathrm{GmbH}$, Germany), each containing the prepared blood samples with a total volume of $7.5 \mathrm{~mL}$. Samples were extracted to perform baseline measurements on protein levels before dividing the cell suspension over the experimental setups. Motion of the cell suspension was driven through a pulsatile flow using a pressure pump (Ibidi $\mathrm{GmbH}$ ). Flow conditions were set at a frequency of $1 \mathrm{~Hz}$ with a peak shear stress and peak pressure of $\sim 1.6 \mathrm{~Pa}$ and $20 \mathrm{mmHg}$, respectively, to mimic physiological pulmonary valvular hemodynamic conditions. Samples of the circulating cell suspension were taken every hour during flow using in-line injection ports (Ibidi $\mathrm{GmbH}$ ) and fixated in formaldehyde (3.7\% v/v; Merck Millipore). These samples were later on used for flow cytometry. After $5 \mathrm{~h}$, the experiment was terminated and the biomaterials were taken out for further analysis. For multiplex ELISA, $300 \mu \mathrm{L}$ of cell suspension was taken and centrifuged for $1500 \mathrm{~g}$ to obtain platelet-rich medium. Plasma was transferred to new tubes and centrifuged for $13,000 \mathrm{~g}$ to obtain platelet-free plasma and snap-frozen immediately afterward. Biomaterial samples were fixed in formaldehyde $(3.7 \% \mathrm{v} / \mathrm{v}$; Merck Millipore) for confocal microscopy and histology or fixated in glutaraldehyde $(2.5 \% \mathrm{v} / \mathrm{v}$; Sigma-Aldrich) for SEM.

\section{Analyses}

Flow cytometry. To assess the composition of the circulating cell population, samples ( $n=4$ per time point) were analyzed with a benchtop flow cytometer (Guava easyCyte 6HT; Merck Millipore). Samples were centrifuged at $200 \mathrm{~g}$ and resuspended in PBS. Debris was excluded using a threshold on the forward scatter signal and at least 20,000 events were counted per sample. Data analysis was performed using Guava Express Pro software (Guava Express Pro; Merck Millipore) in combination with InCyte software (InCyte v2.7; Merck Millipore). Granulocytes were separated from the agranulocytes by gating first on cell size and granularity (forward scatter and side scatter, respectively) and on color in a second gating step. Data were normalized to the amount of circulating cells at the start of the experiment. The best fit was determined for either a line or one-phase decay.

Confocal microscopy. Fixed biomaterial samples $(n=4$ per group) were analyzed with a confocal microscope (TCS SP5X; Leica, Germany) to investigate the distribution of different cell fractions. Granulocytes were visible in red and agranulocytes in green. Scaffold remnants could be visualized through autofluorescence in the blue channel. Samples were immersed in PBS and mounted between two preparation glasses. The specimens were observed with a $10 \times$ objective and Z-stacks were made throughout a depth of $200 \mu \mathrm{m}$. Afterward, a maximum intensity projection was made in Z-direction.

Fluorescence microscopy. To investigate the infiltration depth of the cells into the biomaterials, histology was performed. After confocal microscopy analyses, samples were embedded in Tissue-Tek (Sakura, The Netherlands) and cured gradually in liquid nitrogen vapor. Cryosections of $60 \mu \mathrm{m}$ thickness were cut, embedded in Mowiol (Sigma-Aldrich), and analyzed through fluorescence microscopy (Axiovert 200 M; Zeiss, Germany). Scale bars represent $200 \mu \mathrm{m}$.

Scanning electron microscopy. To investigate cell attachment to the biomaterials, glutaraldehyde-fixed samples were washed twice in PBS and dehydrated in a graded ethanol series, starting from $70 \%(\mathrm{v} / \mathrm{v})$ to $100 \%$ (v/v) with $10 \%$ increments, whereafter the ethanol was allowed to evaporate. Samples were visualized by SEM (Quanta 600F; FEI, The Netherlands) with a $3.0 \mathrm{kV}$ electron beam at low vacuum mode.

Multiplex ELISA. Released cytokine profiles in response to the biomaterials were analyzed using multiplex ELISA. These tests have been performed by the Multiplex core facility of the Laboratory for Translational Immunology of the 
University Medical Center Utrecht in the Netherlands by using an in-house developed and validated multiplex immunoassay based on Luminex Technology. Samples were incubated with antibody-conjugated MagPlex microspheres (BioRad, Hercules) for $1 \mathrm{~h}$ at room temperature with continuous shaking, followed by 1-h incubation with biotinylated antibodies, and 10-min incubation with phycoerythrinconjugated streptavidin diluted in high-performance ELISA (HPE) buffer (Sanquin, The Netherlands). Acquisition was done using a FLEXMAP 3D system controlled with xPONENT software (v 4.1; Luminex). Data were analyzed by five-parametric curve fitting using Bio-Plex Manager software (version 6.1.1; BioRad).

\section{Statistics}

For flow cytometry, data are represented as mean \pm standard error of the mean. Differences in depletion profiles were investigated by using nonlinear regression analysis in terms of a one-phase decay fit with a fixed constraint of $100 \%$ at the start. For multiplex analyses, data are represented as mean \pm standard deviation and analyzed through one-way analysis of variance, followed by Dunnett's multiple comparison correction. Statistical analysis was performed using Prism software (v5.0 GraphPad Software) with results considered significant for $p$ values $<0.05$.

\section{Results}

\section{Blood preparation}

The purity of stained cell fractions was analyzed using flow cytometry. To distinguish granulocytes from agranulocytes, first, cells were gated based on size and granularity (Fig. 2A). Second, events were gated based on fluorescence intensity (Fig. 2B), whereby two peaks could be observed in the agranulocyte fraction (Fig. 2C) and one peak was evidenced in the granulocyte fraction (Fig. 2D). Positive stained events were calculated as a percentage of all events. This resulted in stained events in red of $20.65 \% \pm 6.38 \%$, greenstained events of $26.89 \% \pm 6.99 \%$, or a double staining of $2.29 \% \pm 1.26 \%$. From all the single red events, $86.09 \% \pm$ $5.65 \%$ resided within the granulocyte gate and $6.57 \% \pm 6.49 \%$ within the agranulocyte gate. In case of the single green events, $89.37 \% \pm 7.69 \%$ resided within the agranulocyte gate and $5.91 \% \pm 3.38 \%$ within the granulocyte gate.

\section{Circulating cells}

The circulating cell population was investigated over time through flow cytometry. It appeared that the granulocyte fraction in the bare scaffold samples depleted faster from the circulation compared with DTECs ( $p=0.0022)$ (Fig. 3). In this study, after $60 \mathrm{~min}$, the circulating cells decreased to $46.16 \% \pm 6.47 \%$ of the
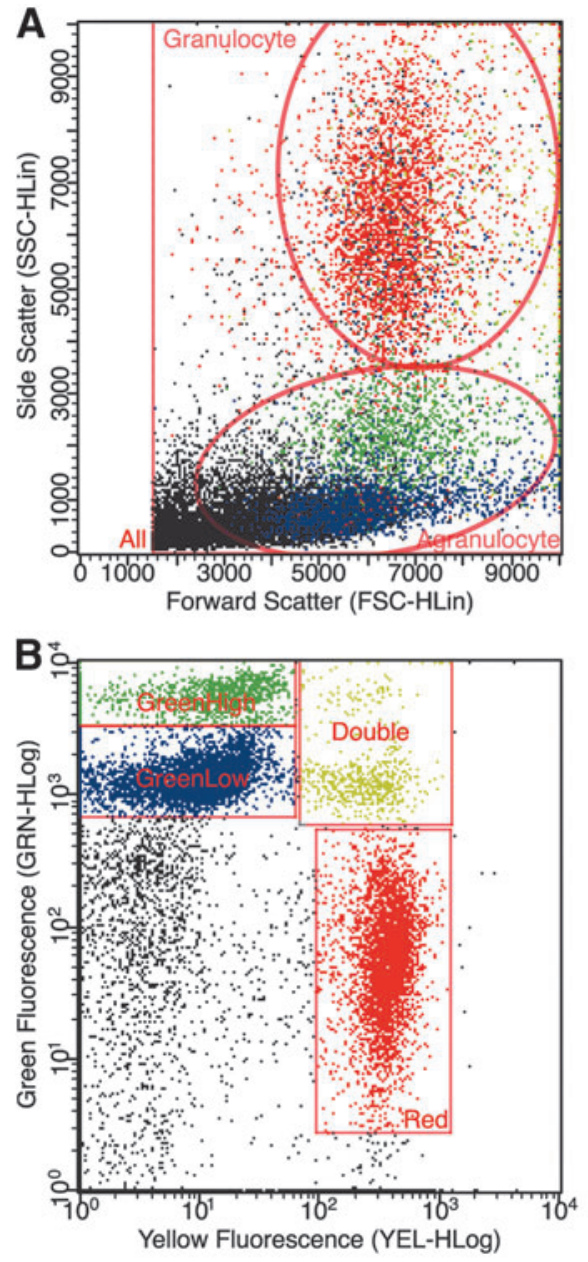
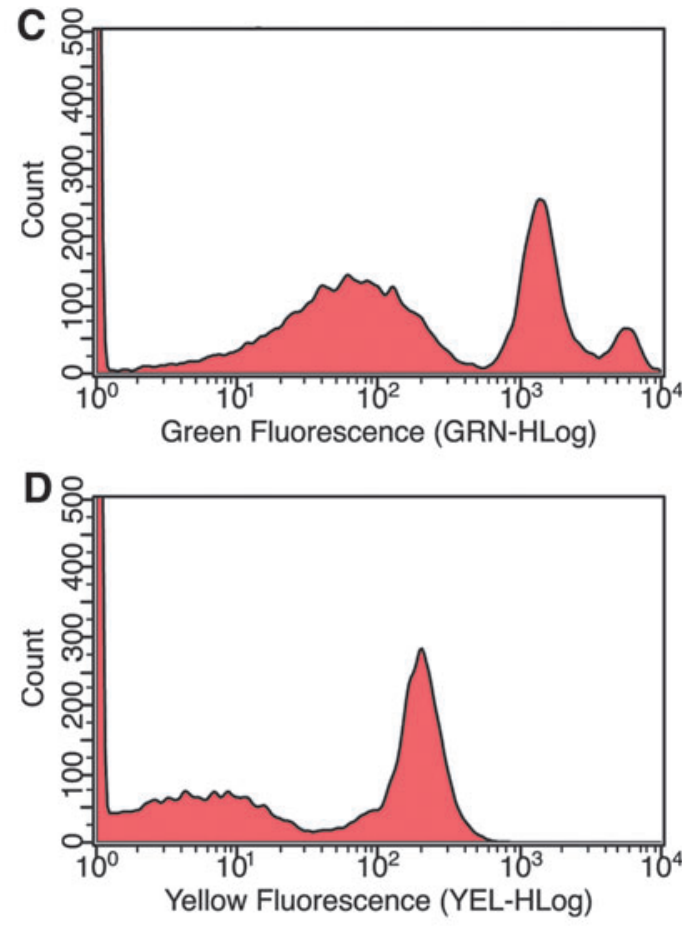

\begin{tabular}{lr} 
E Red & $20.50 \pm 6.38[\%]$ \\
Granulocytes & $86.09 \pm 5.65[\%]$ \\
Agranulocyte & $6.57 \pm 6.49[\%]$ \\
\hline Green & $26.89 \pm 6.99[\%]$ \\
Agranulocyte & $89.37 \pm 7.69[\%]$ \\
Granulocytes & $5.91 \pm 3.38[\%]$ \\
Double & $2.29 \pm 1.26[\%]$
\end{tabular}

FIG. 2. Flow cytometry analyses. Representative side-forward scatter plot, including the applied gates for granulocyte and agranulocyte fractions (A). A representative green-yellow fluorescence plot with a high and low green gate, a yellow gate, and a double stained gate (B). Green fluorescence intensity plot with two distinguishable peaks (C). Yellow fluorescence intensity plot with one clear distinguishable peak (D). The counted color-gated fractions represented as percentage, and the associated cell type discrimination fraction $(\mathbf{E})$. 

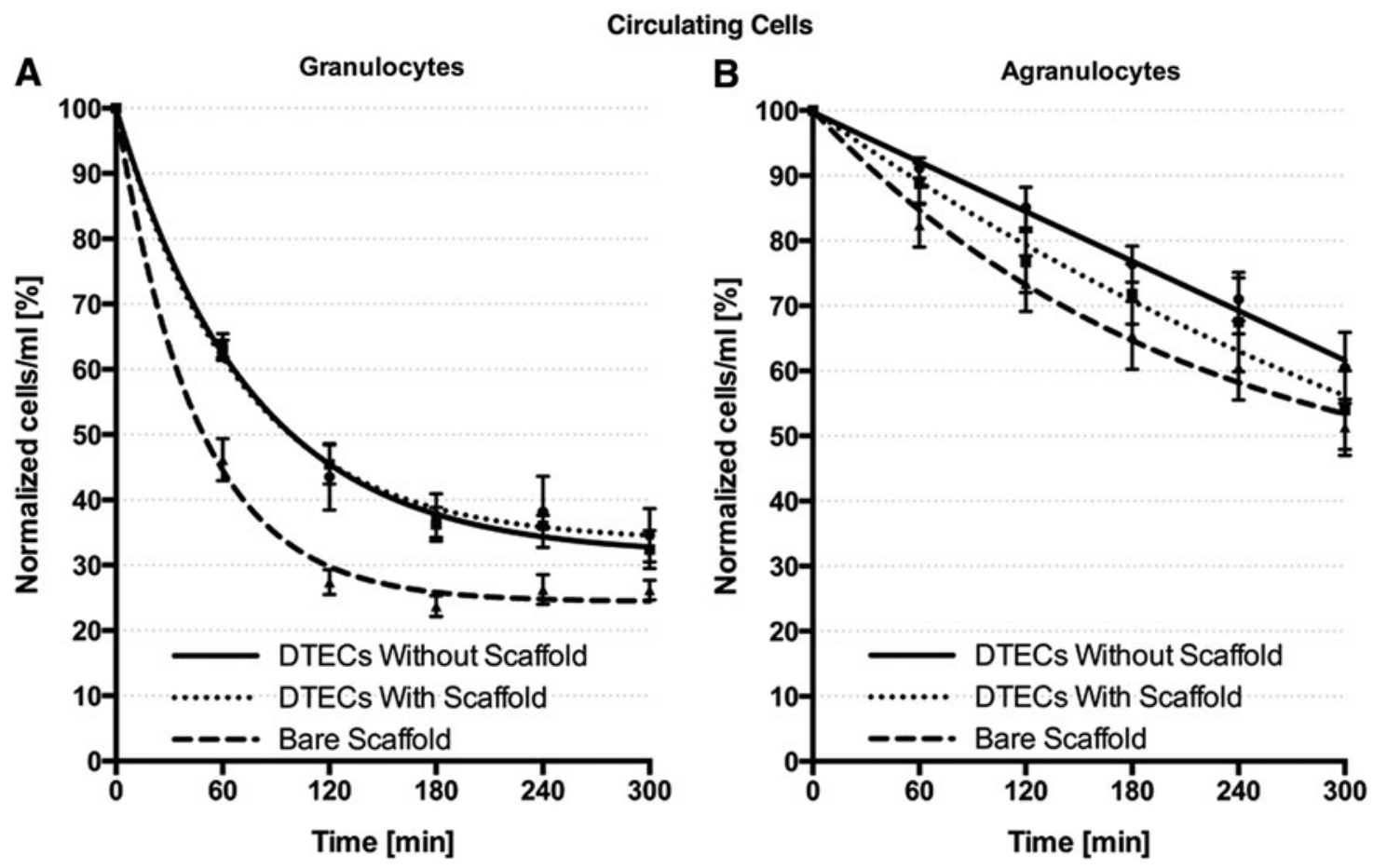

FIG. 3. Circulating cell population. Depletion of circulating cells over the course of the experiment, with fast depletion of the granulocyte fraction (A) compared with the agranulocyte fraction (B) and faster depletion and a lower plateau level of granulocytes in the bare scaffold samples compared with tissue-engineered constructs (A). DTEC, decellularized tissueengineered construct.

initial starting value, being significantly lower compared with both DTECs $(p=0.0017)$. In addition, the plateau level of bare scaffold samples was lower than DTECs $(p=0.0016)$.

\section{Cellular distribution}

Cell distribution was analyzed with confocal microscopy. In all biomaterials, cells were homogeneously distributed over the sample. In the bare scaffold, both granulocytes and agranulocytes were located near the scaffold fibers (Fig. 4A). In the DTEC with scaffold fibers also, both granulocytes and agranulocytes appeared near the fibers, but the agranulocytes were as well observed between the fibers.

\section{Cellular infiltration}

To see to what extent cells actually infiltrated into the biomaterials, cross sections of individual biomaterials were made. In the bare scaffold samples, cells were observed throughout the entire thickness of the biomaterial (Fig. 5A). In the DTECs with scaffold, granulocytes were observed in the core of the biomaterial, whereas the agranulocytes were located at the surface (Fig. 5B). Therefore, it seems that within $5 \mathrm{~h}$, the granulocytes are actually capable of infiltrating into the core of the biomaterial. In case of the DTECs without scaffold, both granulocytes and agranulocytes were located at the surface of the biomaterial. No cellular infiltration was observed in these constructs.

\section{Surface profile}

A surface analysis was performed through SEM. On the bare scaffold samples, cells were located and homogeneously distributed along the individual scaffold fibers (Fig. 6A). On DTECs with scaffold, cells were adhering to the surface of the tissue. Scaffold fibers were nicely embedded into the tissue and not exposed to the surrounding (Fig. 6B). In DTECs without scaffold, cells were observed adhering to the surface (Fig. 6C).

\section{Cytokine release}

After $5 \mathrm{~h}$, the experiment was terminated and the circulating medium was analyzed for the enzyme release profile (Fig. 7). What was observed is that inflammatory regulating cytokines, tumor necrosis factor alpha $(\mathrm{TNF} \alpha)(p=0.0259)$, interleukin (IL)-6 $(p=0.0057)$, and IL- $1 \alpha(p=0.00233)$, in DTECs without scaffold were all upregulated compared with the control levels. The level of monocyte recruiting cytokine monocyte chemotactic protein 1 was upregulated in both DTECs without scaffold $(p=0.0064)$ and DTECs with scaffold ( $p=0.0007)$ compared with the control group. When looking at the protein matrix metalloproteinase-1 (MMP-1), which is associated with active cell infiltration by means of collagen degradation, this protein is significantly upregulated only in DTECs with scaffold material $(p<0.0001)$, indicative of active cellular infiltration into this biomaterial.

\section{Discussion}

DTEHV implantation has shown cell infiltration within hours in previous in vivo experiments. ${ }^{9,10}$ These observations anticipated the possibility to provide regenerative capacity to the bioprosthetic heart valve. Based on histological findings, the infiltrated cells were mainly located in 

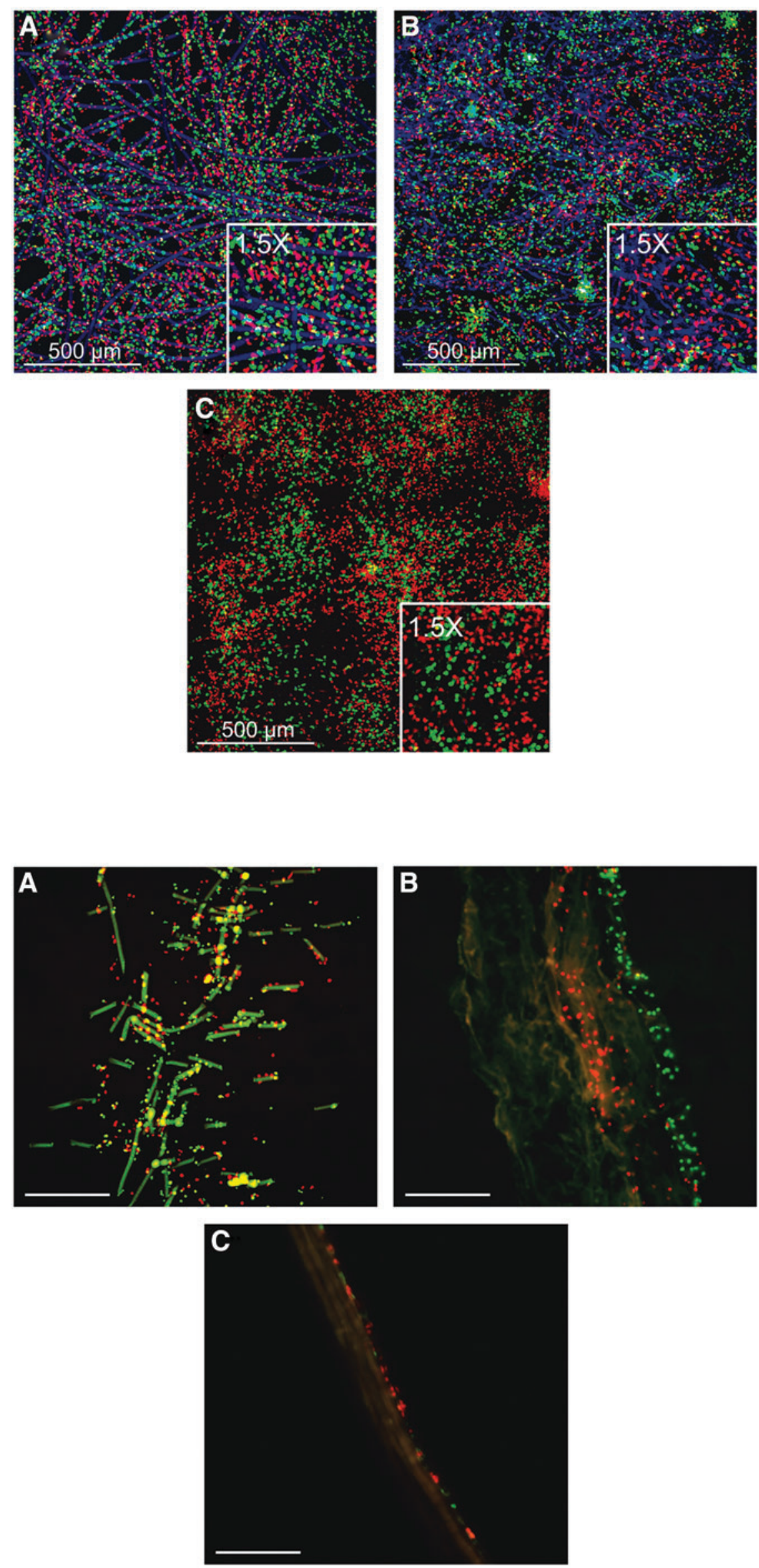

FIG. 4. Cellular distribution. Maximum projection of confocal Z-stacks throughout the different biomaterials. Bare scaffold shows homogeneous cell distribution with both granulocytes and agranulocytes adherent to scaffold fibers (A). Decellularized tissue-engineered constructs with scaffold remnants show the granulocyte fraction in the vicinity of the embedded scaffold fibers (B). The decellularized tissue-engineered constructs without scaffold remnants also show a homogeneous cellular distribution (C). Red cells represent granulocytes, green cells the agranulocytes, and blue the scaffold.

FIG. 5. Cellular infiltration. Representative cross sections of cryosections. Bare scaffold samples show cellular distribution throughout the entire thickness (A). Only granulocyte infiltration in the decellularized tissueengineered constructs with scaffold; a layer of agranulocytes on the surface (B). Both granulocytes and agranulocytes on the surface of decellularized tissue-engineered constructs without scaffold (C). Scale bars indicate $200 \mu \mathrm{m}$. Red indicates the granulocytes and green the agranulocytes. 
FIG. 6. Surface analyses. Scanning electron microscopy images on bare scaffold samples show cells adhering to the scaffold fibers (A). Decellularized tissue-engineered constructs with scaffold remnants show cells on the outside and complete embedding of the scaffold material into the tissue (B). Decellularized tissue-engineered constructs without scaffold reveal a smooth surface with cells adhering (C). Scale bars indicate $100 \mu \mathrm{m}$.
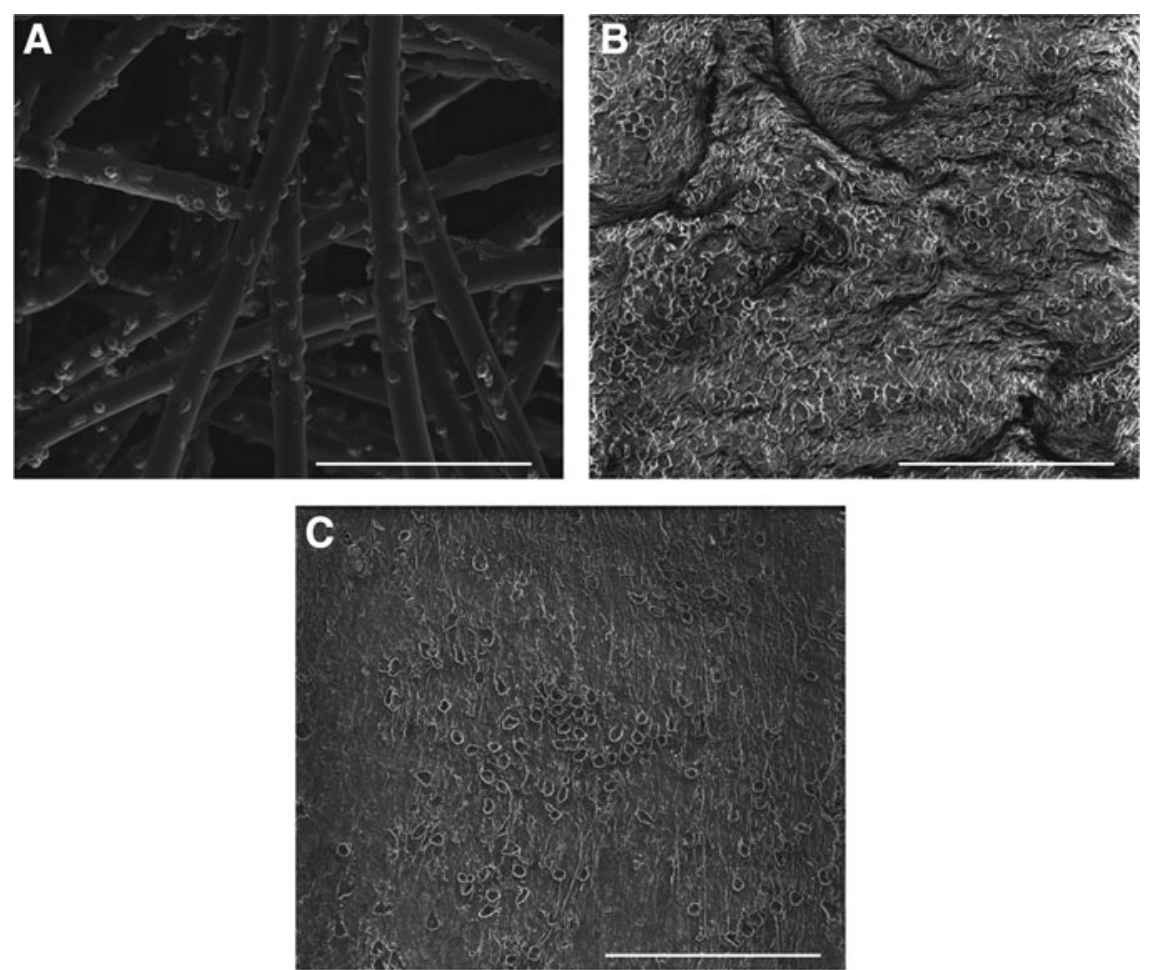

the vicinity of scaffold remnants. This raised the question whether the scaffold material, residing inside the DTEHVs, was responsible for triggering active cell infiltration. In this study, three different biomaterials were produced and tested using a mesofluidic bioreactor system that circulates cells under physiological conditions. DTECs were successfully produced, either with or without scaffold material, and their effects on the circulating cells were compared with the bare scaffold material.

Blood cells were isolated and stained to distinguish between the granulocyte and agranulocyte fractions. Flow cytometry analyses, however, revealed three distinguishable cell populations with one clear peak originating from the granulocyte cell fraction and two clear distinguishable peaks arising from the agranulocyte fraction. Even in the control samples containing only the agranulocyte fraction, two clear distinguishable peaks could be observed (results not shown). The dye used in this experiment relies on an intracellular glutathione S-transferase-mediated reaction to activate fluorescence. Glutathione levels relate to the metabolic activity of eukaryotic and prokaryotic cells and are regulated by mitochondrial activity. ${ }^{20}$ This leads to different glutathione levels between cell types, having higher concentrations in the monocyte population compared with the lymphocyte population. ${ }^{21}$ This might explain why the agranulocyte fraction revealed two distinguishable fluorescent peaks, having a stronger signal for the monocyte fraction indicated in green compared with the lymphocyte fraction indicated in blue (Fig. 2A, B).

Based on flow cytometry analyses after $5 \mathrm{~h}$ of circulation, the agranulocyte population is still above $50 \%$ of the initial concentration (Fig. 3). When comparing this with the granulocyte fraction, it appears that the curve is still in its linear phase when being above its half-time value. There- fore, it can be assumed that the agranulocyte fraction might eventually also decrease in a similar exponential way. However, a 5-h time span might not be sufficient to observe this behavior.

A clear difference in infiltration speed and total amount of cell infiltration was observed in the granulocyte fraction of the bare scaffold sample compared with both DTECs. This can be explained by the porous three-dimensional (3D) open structure of the biomaterial. Since all the individual scaffold fibers are in direct contact with the cells, the total surface area to which cells can adhere is much larger compared with the other biomaterials. Interestingly, no differences in granulocyte depletion were observed between the two types of DTECs. Despite the fact that it appeared that the granulocytes actively infiltrated into the tissue-engineered samples with scaffold, as was shown from the fluorescence cross-section analyses, the total amount of infiltrating cells from this fraction was not significantly larger.

What can be seen from the infiltrated cells in the tissueengineered sample with scaffold (Fig. 4B) is that granulocytes were observed mainly near the scaffold fibers as depicted in blue. This result suggests that the infiltrated cells actively migrate toward the scaffold remnants, which are embedded into the tissue. Granulocyte infiltration into the core of the construct can be seen in Figure 5B; however, in Figure 6B, no scaffold fibers appeared to be present on the outside of the tissue-engineered construct. This might explain that upregulation of MMP-1 was probably secreted by the granulocyte fraction to actively infiltrate the tissue construct.

In this study, the rapid recellularization of granulocytes and the increase of proinflammatory cytokines might not be favorable for tissue regeneration. Future studies are therefore required to investigate which specific cell fraction from 
TNF $\alpha$
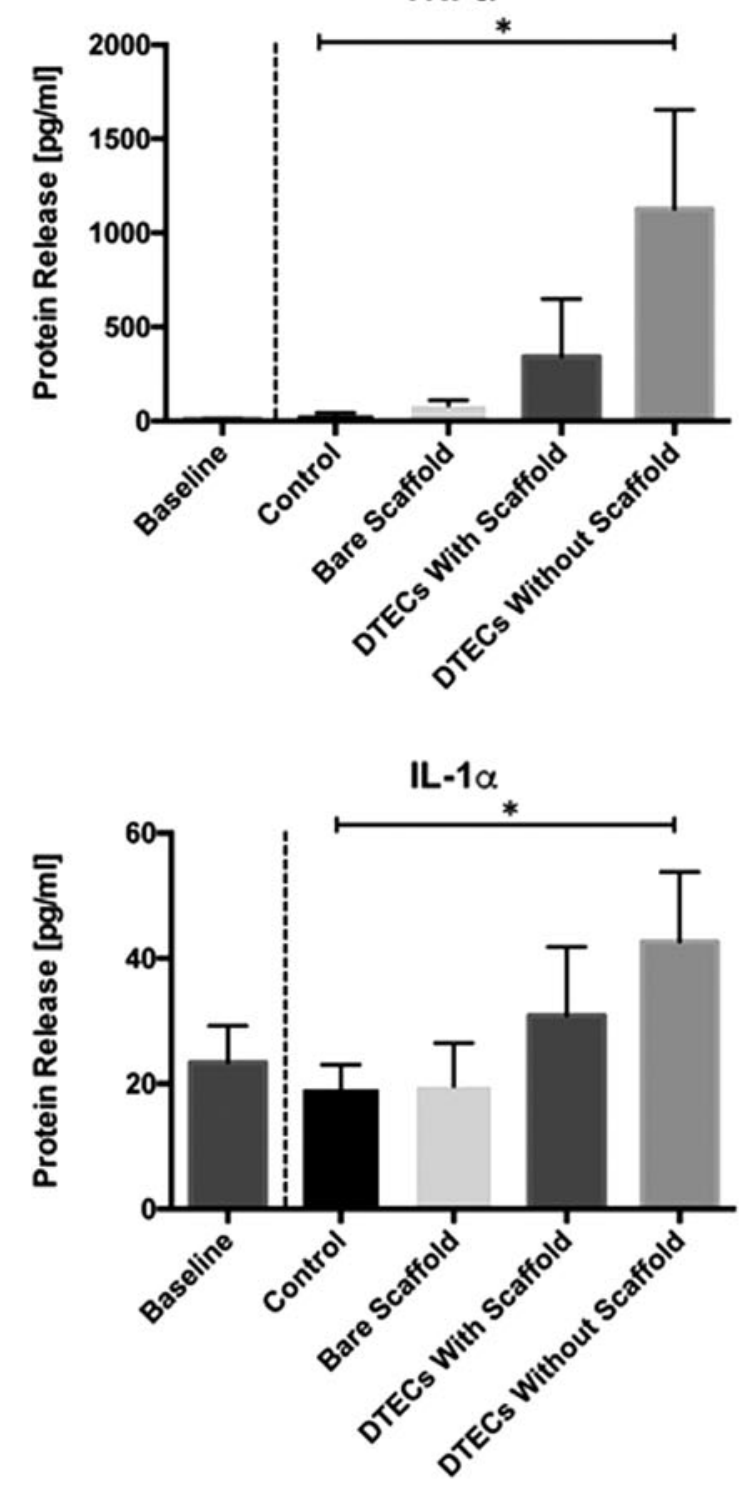

IL-6
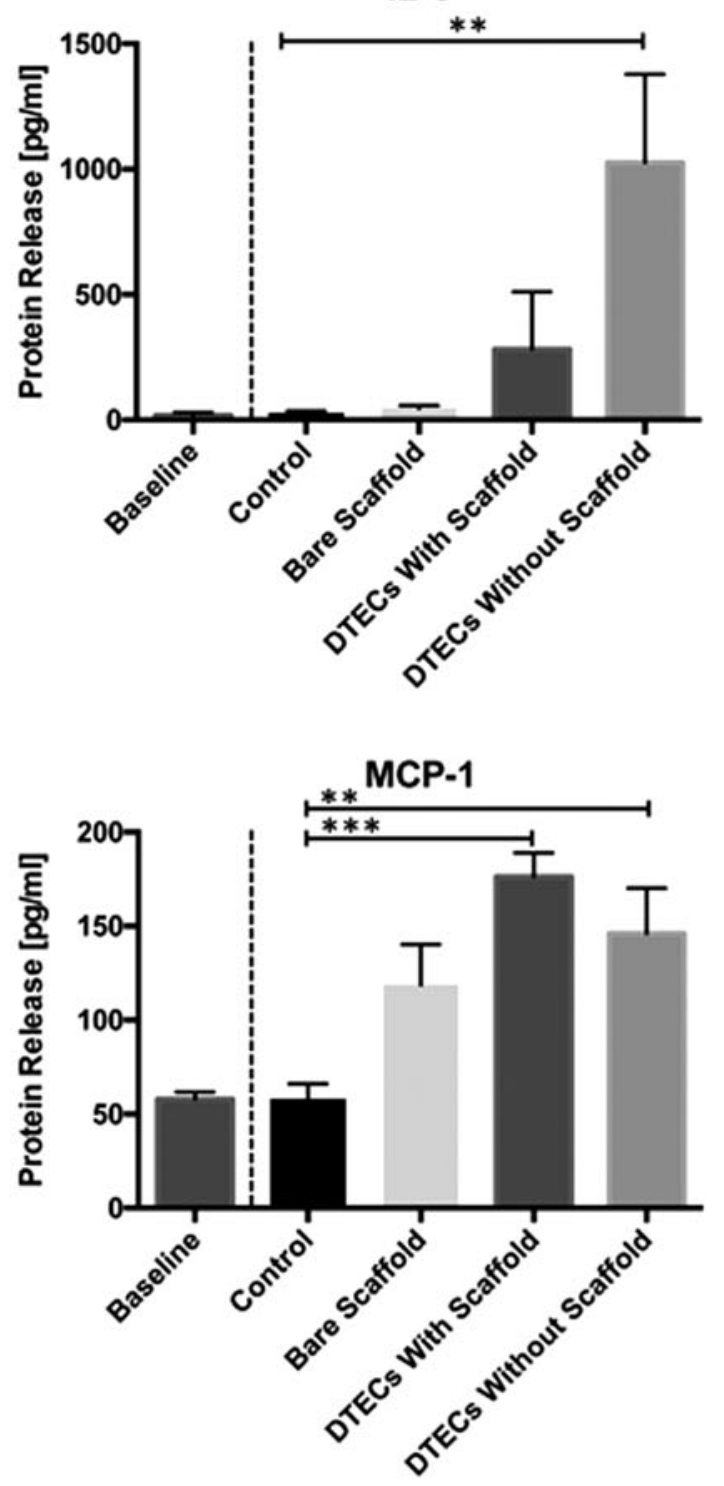

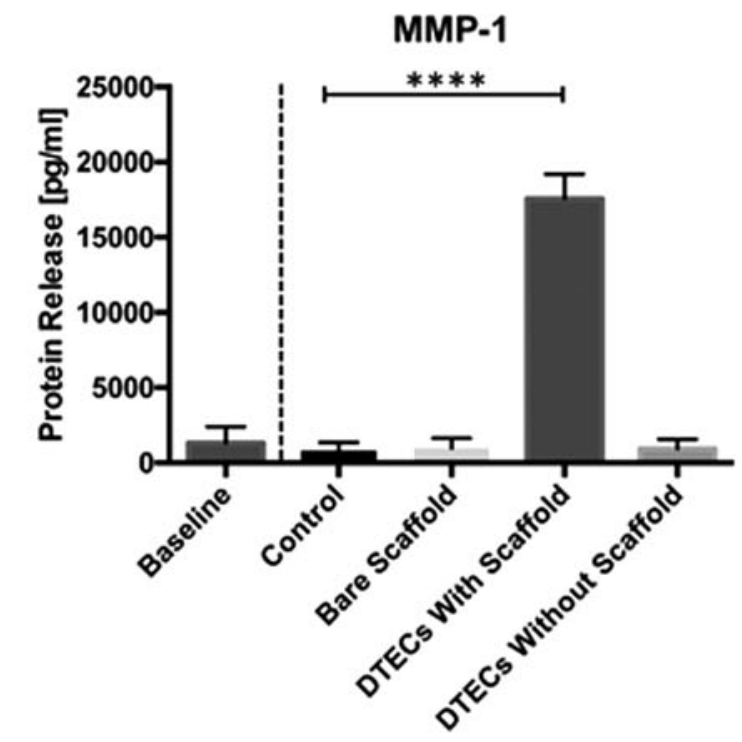

FIG. 7. Cytokine release. Cytokine release profiles after $5 \mathrm{~h}$ of testing. Significant increase of the proinflammatory cytokines, TNF $\alpha$, IL-6, and IL-1 $\alpha$, in the tissue constructs without scaffold remnants. MCP-1 is upregulated in both tissueengineered constructs. MMP-1 is significantly upregulated only in the tissue construct that does contain scaffold remnants, indicative of active cell infiltration. IL, interleukin; TNF $\alpha$, tumor necrosis factor alpha; MCP-1, monocyte chemotactic protein 1; MMP-1, matrix metalloproteinase. 
the granulocyte population infiltrated the DTECs with scaffold remnants and the effect of the first influx of granulocytes on the differentiation and polarization of monocytes and macrophages in the secondary influx of agranulocytes. A possible strategy would be to isolate cell fractions using fluorescence-activated cell sorting, by which cell populations could be stained and tracked accordingly, or even be fully excluding from the study. In this way, a better understanding of a possible priming effect from the granulocytes and the influence on tissue regeneration on the agranulocytes could be further investigated.

The granulocytes infiltrated into the constructs that contained scaffold remnants, but not in those without the scaffold. Therefore, it seems that circulating cells are able to sense the presence of encapsulated remnants. In this study, chemotactic compounds could have been formed during decellularization as no protease inhibitors were used. In addition, the influence of polymer degradation on the local acidity and cell activation might be worthwhile to further investigate. Although it is still not fully understood how these immune cells sense the presence of foreign material, it could be hypothesized that Toll-like receptors might play an important role to detect polymer biodegradable products. ${ }^{22}$ During culture of tissue-engineered constructs over a period of 4 weeks, PGA scaffold starts hydrolyzing after the second week, ${ }^{18}$ but still polymer remnants will be present by the end of the culture procedure. After implantation, ongoing degradation of these remnants could lead to a local $\mathrm{pH}$ drop, making the environment more acidic. ${ }^{23}$ Although it is known that an acidic environment can be beneficial for integrin activation, thereby affecting cellular adhesion and migration, ${ }^{24}$ there is also contradicting literature available describing the impaired effects of a low $\mathrm{pH}$ on granulocyte migration and infiltration. ${ }^{25}$

Despite the effects on cellular migration, changes in extracellular $\mathrm{pH}$ can heavily affect immune activity and result in a diminished release of proinflammatory cytokines when these secreting cells are present in acidic environments. ${ }^{26}$ In this study, proinflammatory cytokine release of IL-1 $\alpha$, IL-6, and $\mathrm{TNF} \alpha$ had a decreasing trend as the amount of scaffold content increased. Therefore, it can be hypothesized that these fast degrading polymers create a local acidic environment that can suppress a proinflammatory response. It has to be mentioned that enzyme secretion over time was not further investigated and is therefore a limitation of this study. How results from these protein release profiles translate to the pediatric application still remains to be evaluated as the adaptive immune system of young children might still be underdeveloped compared with adults. ${ }^{27}$ However, within the $5 \mathrm{~h}$ evaluated in this study, the influence of the adaptive immune response might be negligible as the observations are most likely a direct result of mainly the innate immune response within this time frame. Where various research groups use peripheral blood mononuclear cells to investigate the regenerative response of immune cells to biomaterials, this study highlights the importance of including granulocytes for future in vitro studies.

\section{Conclusion}

This study was aimed at finding the reason why our DTEHVs showed cellular infiltration within hours of im- plantation. An in vitro setup was used to bring blood cells in contact with tissue-engineered biomaterials, either with or without scaffold remnants, and compare them with bare scaffolds. Results show that granulocyte fractions are activated mainly within $5 \mathrm{~h}$. These cells have shown to infiltrate into the core of the construct only in the tissue-engineered samples that do contain scaffold material. Once inside the construct, granulocytes migrate toward the scaffold remnants. Furthermore, MMP-1 is significantly upregulated in the tissue-engineered constructs, including scaffold remnants. These results confirm the hypothesis that scaffold remnants are responsible for early recruitment of blood cells. However, proinflammatory cytokines such as IL- $1 \alpha$, IL-6, and TNF $\alpha$ are significantly upregulated only in the constructs without scaffold remnants. To summarize, scaffold remnants in tissue-engineered constructs can influence the immune response and promote rapid cellular infiltration.

\section{Acknowledgments}

This work was financially supported by the European Union's Seventh Framework Programme (FP7/2007-2013) under grant agreement number 242008 (LifeValve). The authors thank Dr. Maria Sol Cabrera (Biomedical Engineering, Eindhoven University of Technology) for her help with editing the manuscript.

\section{Disclosure Statement}

No competing financial interests exist.

\section{References}

1. Fahed, A.C., Gelb, B.D., Seidman, J.G., and Seidman, C.E. Genetics of congenital heart disease: the glass half empty. Circ Res 112, 707, 2013.

2. Sacks, M.S., Schoen, F.J., and Mayer, J.E., Jr. Bioengineering challenges for heart valve tissue engineering. Annu Rev Biomed Eng 11, 289, 2009.

3. Henaine, R., Roubertie, F., Vergnat, M., and Ninet, J. Valve replacement in children: a challenge for a whole life. Arch Cardiovasc Dis 105, 517, 2012.

4. Fioretta, E.S., Dijkman, P.E., Emmert, M.Y., and Hoerstrup, S.P. The future of heart valve replacement: recent developments and translational challenges for heart valve tissue engineering. J Tissue Eng Regen Med 2016 [Epub ahead of print]; DOI: 10.1002/term.2326.

5. Tedder, M.E., Simionescu, A., Chen, J., Liao, J., and Simionescu, D.T. Assembly and testing of stem cell-seeded layered collagen constructs for heart valve tissue engineering. Tissue Eng Part A 17, 25, 2011.

6. Miller, D.V., Edwards, W.D., and Zehr, K.J. Endothelial and smooth muscle cell populations in a decellularized cryopreserved aortic homograft (SynerGraft) 2 years after implantation. J Thorac Cardiovasc Surg 132, 175, 2006.

7. Woo, J.S., Fishbein, M.C., and Reemtsen, B. Histologic examination of decellularized porcine intestinal submucosa extracellular matrix (CorMatrix) in pediatric congenital heart surgery. Cardiovasc Pathol 25, 12, 2016.

8. Kheradvar, A., Groves, E.M., Dasi, L.P., Alavi, S.H., Tranquillo, R., Grande-Allen, K.J., et al. Emerging trends in heart valve engineering: part I. Solutions for future. Ann Biomed Eng 43, 833, 2015. 
9. Driessen-Mol, A., Emmert, M.Y., Dijkman, P.E., Frese, L., Sanders, B., Weber, B., et al. Transcatheter implantation of homologous "Off-the-Shelf" tissue-engineered heart valves with self-repair capacity. J Am Coll Cardiol 63, 1320, 2014.

10. Weber, B., Dijkman, P.E., Scherman, J., Sanders, B., Emmert, M.Y., Grünenfelder, J., et al. Off-the-shelf human decellularized tissue-engineered heart valves in a nonhuman primate model. Biomaterials 34, 7269, 2013.

11. Badylak, S.F., and Gilbert, T.W. Immune response to biologic scaffold materials. Semin Immunol 20, 109, 2008.

12. Badylak, S.F., Valentin, J.E., Ravindra, A.K., McCabe, G.P., and Stewart-Akers, A.M. Macrophage phenotype as a determinant of biologic scaffold remodeling. Tissue Eng Part A 14, 1835, 2008.

13. Brown, B.N., Ratner, B.D., Goodman, S.B., Amar, S., and Badylak, S.F. Macrophage polarization: an opportunity for improved outcomes in biomaterials and regenerative medicine. Biomaterials 33, 3792, 2012.

14. Smits, A.I.P.M., Driessen-Mol, A., Bouten, C.V.C., and Baaijens, F.P.T. A mesofluidics-based test platform for systematic development of scaffolds for in situ cardiovascular tissue engineering. Tissue Eng Part C Methods 18, 475, 2012.

15. Mol, A., van Lieshout, M.I., Dam-de Veen, C.G., Neuenschwander, S., Hoerstrup, S.P., Baaijens, F.P.T., et al. Fibrin as a cell carrier in cardiovascular tissue engineering applications. Biomaterials 26, 3113, 2005.

16. Schnell, A.M., Hoerstrup, S.P., Zund, G., Kolb, S., Sodian, R., Visjager, J.F., et al. Optimal cell source for cardiovascular tissue engineering: venous vs. aortic human myofibroblasts. Thorac Cardiovasc Surg 49, 221, 2001.

17. Sanders, B., Loerakker, S., Fioretta, E.S., Bax, D.J.P., Driessen-Mol, A., Hoerstrup, S.P., et al. Improved geometry of decellularized tissue engineered heart valves to prevent leaflet retraction. Ann Biomed Eng 44, 1061, 2016.

18. van Vlimmeren, M.A.A., Driessen-Mol, A., Oomens, C.W.J., and Baaijens, F.P.T. An in vitro model system to quantify stress generation, compaction, and retraction in engineered heart valve tissue. Tissue Eng Part C Methods 17, 983, 2011.

19. Dijkman, P.E., Driessen-Mol, A., Frese, L., Hoerstrup, S.P., and Baaijens, F.P.T. Decellularized homologous tissue- engineered heart valves as off-the-shelf alternatives to xeno- and homografts. Biomaterials 33, 4545, 2012.

20. Lash, L.H. Mitochondrial glutathione transport: physiological, pathological and toxicological implications. Chem Biol Interact 163, 54, 2006.

21. Scott, R.B., Collin, J.M., Matin, S., White, F., and Swerdlow, P.S. Simultaneous measurement of neutrophil, lymphocyte, and monocyte glutathione by flow cytometry. J Clin Lab Anal 4, 324, 1990.

22. Shokouhi, B., Coban, C., Hasirci, V., Aydin, E., Dhanasingh, A., Shi, Y., et al. The role of multiple toll-like receptor signalling cascades on interactions between biomedical polymers and dendritic cells. Biomaterials 31, 5759, 2010.

23. Agrawal, C.M., and Athanasiou, K.A. Technique to control $\mathrm{pH}$ in vicinity of biodegrading PLA-PGA implants. J Biomed Mater Res 38, 105, 1997.

24. Paradise, R.K., Lauffenburger, D.A., and Van Vliet, K.J. Acidic extracellular $\mathrm{pH}$ promotes activation of integrin $\alpha(v) \beta(3)$. PLoS One 6, e15746, 2011.

25. Rotstein, O.D., Fiegel, V.D., Simmons, R.L., and Knighton, D.R. The deleterious effect of reduced $\mathrm{pH}$ and hypoxia on neutrophil migration in vitro. J Surg Res 45, 298, 1988.

26. Lardner, A. The effects of extracellular $\mathrm{pH}$ on immune function. J Leukoc Biol 69, 522, 2001.

27. Pettengill, M.A., van Haren, S.D., and Levy, O. Soluble mediators regulating immunity in early life. Front Immunol 5, 457, 2014.

\author{
Address correspondence to: \\ Bart Sanders, PhD \\ Department of Biomedical Engineering \\ Eindhoven University of Technology \\ P.O. Box 513 \\ 5600 MB Eindhoven \\ The Netherlands \\ E-mail: b.sanders@tue.nl \\ Received: November 15, 2016 \\ Accepted: March 15, 2017 \\ Online Publication Date: April 14, 2017
}

